\title{
Evaluation of Left Atrial Function by the Functional Volume Change Curve Derived From Doppler Flow Spectra
}

\author{
Fumiaki Nakao, MD*; Yuichiro Wasaki, MD; Masayasu Kimura, MD*; \\ Takahiro Iwami, MD; Hiroshi Iida, MD; Takatoshi Wakeyama, MD; \\ Toshiro Miura, MD*; Hiroshi Ogawa, MD; Masunori Matsuzaki, MD*
}

\begin{abstract}
The objective of this study was to clarify the left atrial (LA) reservoir and booster pump function in patients with left ventricular (LV) diastolic dysfunction. To determine LA reservoir and booster pump function, a new algorithm to determine LA functional volume change curve (FVC) was developed from Doppler flow spectra of pulmonary venous flow and LV inflow by transthoracic echocardiography in 110 patients. Patients were classified into normal $(\mathrm{N})$, and abnormal $(\mathrm{AB})$ and pseudonormal $(\mathrm{PN})$ groups on the basis of their Doppler flow patterns. From the indices of FVC, atrial reservoir volume (ARV), passive emptying volume (PEV) and active emptying volume (AEV) were obtained. ARV/stroke volume (SV) was increased in the $\mathrm{AB}$ group, but decreased in the PN group compared with $\mathrm{N}(\mathrm{N}, 0.61 \pm 0.09 ; \mathrm{AB}, 0.73 \pm 0.10 ; \mathrm{PN}, 0.52 \pm 0.13, \mathrm{p}<0.05)$. PEV/SV was significantly decreased in $\mathrm{AB}$, but increased in $\mathrm{PN}$ compared with $\mathrm{N}(\mathrm{N}, 0.27 \pm 0.07 ; \mathrm{AB}, 0.19 \pm 0.07$; PN, 0.31 \pm 0.18 , $\mathrm{p}<0.05)$. AEV/SV was significantly increased in $\mathrm{AB}$, but decreased in PN compared with $\mathrm{N}(\mathrm{N}, 0.41 \pm 0.08 ; \mathrm{AB}$, $0.56 \pm 0.10 ; \mathrm{PN}, 0.26 \pm 0.19, \mathrm{p}<0.05$ ). Thus, in patients with an abnormal relaxation pattern, the LA reservoir and booster pump function are augmented, but in patients with a pseudonormal pattern, both LA reservoir and booster pump function are deteriorated, suggesting a vulnerability to pulmonary congestion. (Jpn Circ J 2001; 65: $953-957$ )
\end{abstract}

Key Words: Doppler echocardiography; Left atrial function; Left ventricular diastolic function; Volume curve

$\coprod$ eft atrial (LA) function can be evaluated by measuring pressure and volume, constructing the pressurevolume relationship and the dimensional changes of the LA can replace LA volume!-8 LA function has 3 components: reservoir, conduit and booster pump functions, $5,6,10$ The reservoir function indicates the storage capacity for pulmonary venous (PV) return by LA distension during ventricular systole. During ventricular diastole, the LA works as a conduit for blood flow from the PV to the left ventricle (LV). The booster pump function indicates the filling blood volume to the LV during atrial systole. Recently, it is shown that atrial natriuretic peptide (ANP) synthesis is activated by the stimulus of atrial stretch, and the booster pump function of LA is strongly related to the release of ANP! ${ }^{1,12}$ Therefore, it is important to estimate the LA function in detail to understand the hemodynamic status of patients with heart failure.

In spite of its importance, it is difficult to estimate LA function, particularly the 3 components of LA function, because of the difficulty in determining the change in LA volume correctly. Thus, we developed a new method for measuring the LA volume change independent of the LA shape using Doppler flow spectra. PV flow (PVF) velocity and LV inflow (LVIF) velocity were measured by Doppler echocardiography, and from these flow velocity data the

(Received December 18, 2000; revised manuscript received July 19, 2001; accepted August 9, 2001)

Department of Cardiology, Tokuyama Central Hospital and *Department of Cardiovascular Medicine, Yamaguchi University School of Medicine, Yamaguchi, Japan

Mailing address: Fumiaki Nakao, MD, Department of Cardiovascular Medicine, Yamaguchi University School of Medicine, 1-1-1 MinamiKogushi, Ube, Yamaguchi 755-8505, Japan
LA functional volume change curve (FVC) was constructed by a newly developed algorithm described in detail in the Methods section. Using the FVC, we evaluated LA reservoir and booster pump functions, respectively, in patients with normal and abnormal LV diastolic function.

\section{Methods}

\section{Study Patients}

The 110 consecutive patients (male 68, female 42) who did not have mitral regurgitation were enrolled in this study. The mean age of the population was $61.6 \pm 12.5$ years (range, 26-86). The following heart diseases were involved: coronary artery disease, 42; myocarditis, 11; hypertrophic cardiomyopathy, 4; dilated cardiomyopathy, 4; aortic regurgitation, 2; ventricular septal defect, 2; sick sinus syndrome, 1. Thirty-two patients had no heart disease, 4 had respiratory disease and 8 had undergone hemodialysis.

\section{Transthoracic Echocardiography}

Echocardiography was performed using ALOKA SSD2200 with a $2.5 \mathrm{MHz}$ probe in the left lateral decubitus position during quiet respiration. The variability of the R-R interval never exceeded $\pm 5 \% !^{13}$ To record LVIF, the sample volume was placed at the mitral tip in the left anterior oblique-like view! 14,15 For PVF, the color Doppler method was used to identify the PV orifice and the sample volume was placed at the right or left upper PV within $15 \mathrm{~mm}$ of its orifice in either the 4- or 2-chamber view! ${ }^{15} 16$ Standard $\mathrm{M}$ - and B-mode echocardiography was recorded and \% fractional shortening (\%FS) and stroke volume (SV) were calculated from M-mode echocardiography. 


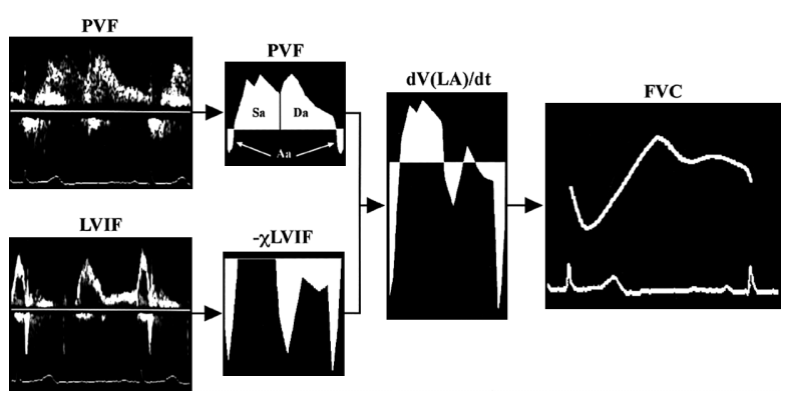

Fig 1. Schema of the composition of the left atrial functional volume change curve (FVC) derived from Doppler flow spectra. PVF, pulmonary venous flow; LVIF, left ventricular inflow; dV(LA)/dt, the first derivative of left atrial volume change; $X$, constantX .

\section{Computation of FVC}

Because the LVIF pattern is similar to the pattern of the first derivative of LV volume change during ventricular diastole, LV volume change can be predicted by LVIF!7 We applied this concept to the LA volume change during 1 cardiac cycle. Theoretically, the inflow volume to the LA and its outflow volume during 1 cardiac cycle are equal. Instantaneous differences between the inflow and outflow volumes indicate the first derivative of LA volume change $(\mathrm{dV}(\mathrm{LA}) / \mathrm{dt})$. Thus, the algorithm to integrate the difference between LA inflow and outflow gives the FVC.

The schema of the computation of the FVC is shown in Fig 1. When we assume that the mitral annular and PV diameters are unchanged during ${ }^{1}$ cardiac cycle and that the $4 \mathrm{PV}$ flow patterns are similar, the following equation is obtained: mitral valve annular area $\times$ the velocity-time integral (VTI) during a cardiac cycle of LVIF $=$ the sum of $4 \mathrm{PV}$ cross-sectional areas $\times$ VTIs during a cardiac cycle of PVF.

With a constantX $=$ mitral valve annular area/the sum of $4 \mathrm{PV}$ cross-sectional areas, the formula becomes as follows: $X$ VTI of LVIF $=$ VTI of PVF.

The instantaneous difference between PVF and X LVIF produces $\mathrm{dV}(\mathrm{LA}) / \mathrm{dt}$. Then, we computed the integrals of $\mathrm{dV}(\mathrm{LA}) \mathrm{dt}$, which produces the FVC.

The VTI of PVF during ventricular systole (Sa) was measured from the onset of the forward flow to the onset of the diastolic flow, which was defined as the nadir between systolic and diastolic waves. The VTI of PVF during ventricular diastole (Da) was measured from the nadir between systolic and diastolic waves to the termination of the diastolic forward wave. The VTI of PVF during atrial systole (Aa) was measured from the onset up to the termination of the backward flow. Atrial reservoir volume (ARV), which was the difference between LA maximum and minimum volumes, was calculated with the following formula ${ }^{16}: \mathrm{ARV}=\mathrm{SV} \times \mathrm{Sa} /(\mathrm{Sa}+\mathrm{Da}-\mathrm{Aa})$.

From the FVC, we calculated the passive emptying volume (PEV), which represents the decreased volume from the LA with LV early diastolic filling, and the active emptying volume (AEV), which represents the decreased volume from the LA by LA contraction (Fig 2). We evaluated LA function by ARV, PEV, AEV, and their values corrected by SV $5,6,9,10,18$

NIH image V1.61 (Wayne Rasband, National Institutes of Health), image-processing software ${ }^{19}$ was used to obtain the FVC. We imported PVF and LVIF spectra during 1 cardiac cycle from the peak of the R wave on the ECG into

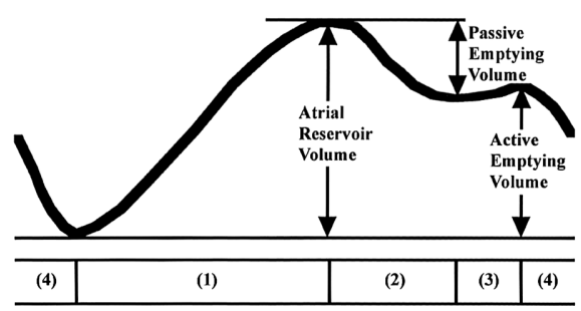

Fig 2. Left atrial functional volume change curve divided into 4 phases: (1) an active filling phase, (2) a passive emptying phase, (3) a diastasis, (4) an active emptying phase.

the computer. By NIH image loading the FVC calculation macro, the pixels of the 2 spectra were counted and the FVC was composed semi-automatically.

Comparison With Aortic-LA M-Mode Echocardiography

The correlation between the LA volume curve and relative changes of the aortic posterior wall (PAW) motion on aortic-left atrial M-mode echocardiography is known ${ }^{20,21}$ LA volume can be calculated from the M-mode LA dimension (LAD) using the curvilinear regression equation $(\mathrm{y}=$ $1.4 \mathrm{x}^{3}+23 ; \mathrm{y}=\mathrm{LA}$ volume $(\mathrm{ml}), \mathrm{x}=\mathrm{M}$-mode LAD $\left.(\mathrm{mm})\right)^{22}$ In 16 patients (female, 4; male, 12; mean age, $60.8 \pm 15.2$ years; coronary artery disease, 13 ; dilated cardiomyopathy, 2; aortic regurgitation, 1), the correlation of ARV, PEV and AEV between the FVC and LA volume calculated from Mmode LAD was examined by simple regression analysis. A p value less than 0.05 was considered statistically significant.

\section{LA Function}

Patients were divided into 3 groups on the baisis of the ratio of peak early filling velocity to peak atrial systolic velocity (E/A) in LVIF, the deceleration time of early filling flow (DcT) in LVIF, and the ratio of peak systolic velocity to peak diastolic velocity (S/D) in PVF. When E/A $\geq 1.0$, DcT $\geq 140 \mathrm{~ms}$, and $\mathrm{S} / \mathrm{D} \geq 1.0$, patients were classified as normal $(\mathrm{N})$, when $\mathrm{E} / \mathrm{A}<1.0$, patients were classified as abnormal (AB), and when E/A $\geq 1.0$, DcT $<140 \mathrm{~ms}$, S/D $<1.0$, patients were classified as pseudonormal $(\mathrm{PN})^{23-25}$ ARV/SV, PEV/ARV, AEV/ARV, PEV/SV, and AEV/SV were compared among the 3 groups.

Using one-way ANOVA followed by Scheffe's F test, patient characteristics and the echocardiographic indices of each group were compared. Values were expressed as mean $\pm S D$. A $p$ value less than 0.05 was considered significant.

\section{Results}

FVC

The FVC shows a similar pattern to the LA volume curve shown in previous studies ${ }^{3-6,18}$ and could be divided into 4 phases as described previously5: an active filling phase in which the LA volume increased with LA relaxation and LV contraction; a passive emptying phase in which the LA volume decreased with LV early diastolic filling; a diastasis in which the LA volume changed slightly; and an active emptying phase in which the LA volume decreased with LA contraction (Fig 2).

Validity of FVC

Fig 3 is representative curves of the FVC and the PAW 
A
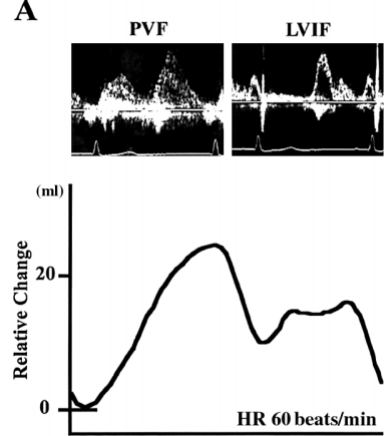

B
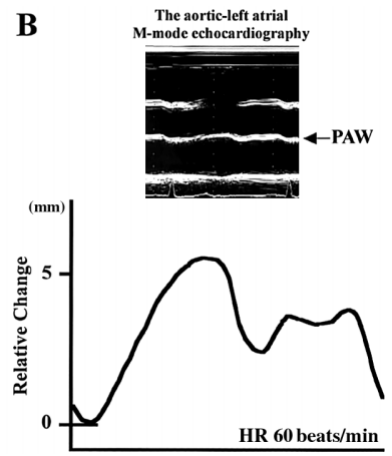

Fig 3. Representative left atrial functional volume change curve derived from Doppler flow spectra (A) and the posterior aortic wall motion (B). PVF, pulmonary venous flow; LVIF, left ventricular inflow; PAW, aortic posterior wall; HR, heart rate.

motion; the 2 curves showed similar patterns. Fig 4A shows the correlation between the ARV derived from the FVC and that from the M-mode LAD, Fig 4B shows the correlation between the PEV derived from the FVC and that from the M-mode LAD, and Fig 4C shows the correlation between the AEV derived from the FVC and that from the M-mode LAD. Significant positive correlations were observed in ARV, PEV and AEV.

\section{Comparison of the Parameters of LA Function}

Table 1 shows the patient characteristics and echocardiogaphic parameters of the 3 groups. The mean age of the AB group was higher than that of the $\mathrm{N}$ group, but other parameters were not significantly different between the $\mathrm{N}$ and the $\mathrm{AB}$ group. The heart rate and the LA dimension in the PN group were higher than those in the $\mathrm{N}$ and $\mathrm{AB}$ groups, and the $\% \mathrm{FS}$ in the PN group was significantly less than that in the $\mathrm{N}$ and the $\mathrm{AB}$ groups.

Representative FVCs of the $\mathrm{N}, \mathrm{AB}$, and $\mathrm{PN}$ groups are shown in Fig 5. Fig 6 shows the indices of the FVC in the 3

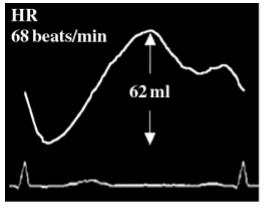

N group

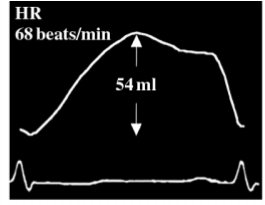

AB group

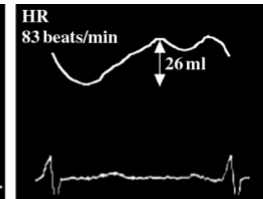

PN group
Fig 5. Representative left atrial functional volume change curve in normal $(\mathrm{N})$, abnormal $(\mathrm{AB})$, and pseudonormal $(\mathrm{PN})$ groups. $\mathrm{HR}$, heart rate.
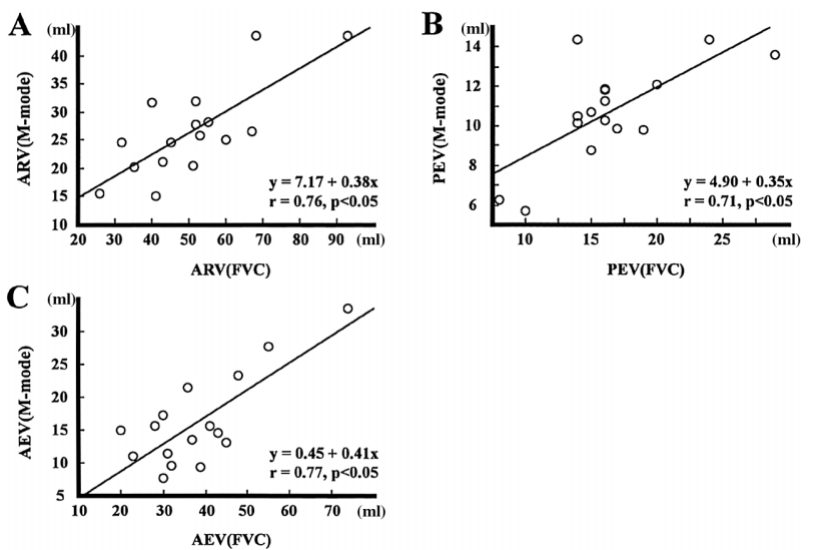

Fig 4. Correlation of the parameters derived from Doppler flow spectra and the M-mode left atrial dimension. FVC, left atrial functional volume change curve derived from Doppler flow spectrums; $\mathrm{ARV}$, atrial reservoir volume; PEV, passive emptying volume; AEV, active emptying volume.

groups. ARV/SV, AEV/ARV and AEV/SV were significantly greater in the $\mathrm{AB}$ group compared with the $\mathrm{N}$ group, whereas those of the PN group were significantly less than those of the $\mathrm{N}$ group. PEV/ARV and PEV/SV were significantly greater in the $\mathrm{PN}$ group that in $\mathrm{N}$ group, whereas that
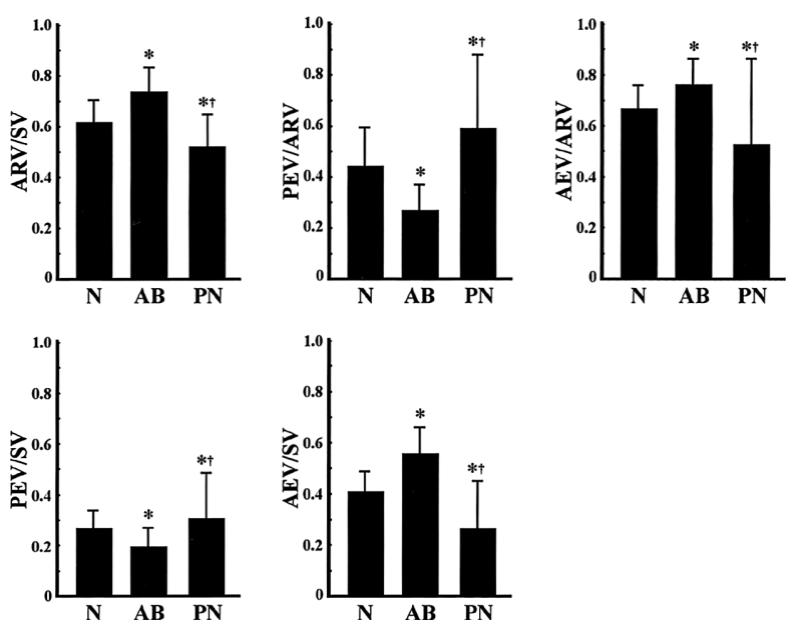

Fig 6. Indices of left atrial function. Values are expressed as mean \pm $\mathrm{SD}$. ${ }^{*} \mathrm{p}<0.05$ vs $\mathrm{N}$ group, ${ }^{\dagger} \mathrm{p}<0.05$ vs $\mathrm{AB}$ group. $\mathrm{N}$, normal; $\mathrm{AB}$, abnormal relaxation; PN, pseudonormal; ARV, atrial reservoir volume; SV, stroke volume; PEV, passive emptying volume; AEV, active emptying volume.

Table 1 Patient Characteristics and Echocardiogaphic Data

\begin{tabular}{lccc}
\hline \hline & $\begin{array}{c}\text { N group } \\
(n=32)\end{array}$ & $\begin{array}{c}\text { AB group } \\
(n=67)\end{array}$ & $\begin{array}{c}P N \text { group } \\
(n=11)\end{array}$ \\
\hline Age (years) & $50.2 \pm 9.0$ & $67.3 \pm 9.8^{*}$ & $58.6 \pm 13.0$ \\
Gender $($ M:F) & $16: 16$ & $46: 21$ & 6.5 \\
Heart rate $($ beats/min $)$ & $63.4 \pm 9.1$ & $67.9 \pm 11.3$ & $90.7 \pm 22.0^{* \dagger}$ \\
LVEDD $(\mathrm{mm})$ & $47.0 \pm 6.5$ & $48.7 \pm 7.0$ & $48.3 \pm 8.3$ \\
\%FS $(\%)$ & $37.5 \pm 9.7$ & $33.1 \pm 10.3$ & $18.3 \pm 5.8^{* \dagger}$ \\
LAD $(\mathrm{mm})$ & $33.0 \pm 4.3$ & $33.7 \pm 4.0$ & $41.1 \pm 4.3^{* \dagger}$ \\
\hline
\end{tabular}

Values are expressed as mean $\pm S D$ or number of patients. ${ }^{*} p<0.05$ vs $N$ group, ${ }^{\dagger} p<0.05$ vs AB group. $N$, normal; AB, abnormal relaxation; PN, pseudonormal; LVEDD, left ventricular end-diastolic dimension; \%FS, \% fractional shortening; LAD, left atrial dimension. 
in $\mathrm{AB}$ group was significantly less than that in $\mathrm{N}$ group.

\section{Discussion}

In order to estimate LA function, the reservoir, conduit and booster pump functions should be separately estimated. For this purpose, it is important to know LA volume change curve and this study demonstrates that the FVC can be composed from Doppler signals, such as PVF and LVIF, and that it was a valid means of indicating LA volume change. Using parameters from the FVC, we then compared the LA function in normal, abnormal and pseudonormal LV relaxation groups defined by LVIF and PVF patterns. Compared with the normal group, the abnormal relaxation group showed increased LA reservoir and booster pump function indicated by ARV/SV and AEV/SV. On the other hand, the pseudonormal group had deteriorated LA reservoir and booster pump functions.

These results indicate that the abnormal relaxation group had a compensated increase in LA reservoir and booster pump function, which may assist in alleviating pulmonary congestion. On the other hand, the pseudonormal group had impaired LA reservoir and booster pump function, which may explain their vulnerability to pulmonary congestion, especially during tachycardia.

\section{Methodological Considerations}

In this study, to calculate the FVC, the PVF obtained from $1 \mathrm{PV}$ instead of 4 was used. Earlier PVF studies reported that the VTIs of PVF between the right upper and left upper PV were not significantly different in the systolic and diastolic waves 26 It has also been reported that ARV/SV in the left upper PV and right upper PV is similar!6 Thus, it is feasible to assume that the flow pattern of $1 \mathrm{PV}$ represents the flow pattern of $4 \mathrm{PVs}$ and it was assumed in this study that PV cross-sectional area did not change during 1 cardiac cycle. Although changes in PV shape and crosssectional area during 1 cardiac cycle has been reported, 13,27 the calculated PV volumetric spectrum (product of PV cross-sectional area and PVF spectrum) could be superimposed over the original PVF spectrum $!^{3}$ Thus, it is reasonable to assume that PV cross-sectional area is constant for calculating the FVC.

It was also assumed that the mitral valve annular area did not change during ventricular diastole, although it has been shown that mitral valve annular area changes in shape and size during 1 cardiac cycle; however, the change is gradual and up to $12 \%$ from early to late diastole 28 Therefore, it is possible to assume that the mitral valve annular area is constant during ventricular diastole for the purposes of calculating the FVC.

\section{Comparison With Other Methods of Measuring the LA Volume Curve}

Compared with other invasive and non-invasive calculation of the LA volume curve, such as left atriography during cardiac catheterization, digital subtraction angiography and gated radionuclide atriography, the FVC derived from Doppler signals is conveniently obtained and non-invasive. The PAW motion and the M-mode LAD may not accurately reflect the LA volume change when there is deformation of the aortic root or LA.

\section{Study Limitations}

When significant mitral regurgitation exists, accurate measurement of inflow and outflow is disturbed and the FVC can not be correctly calculated. Without the simultaneous recording of LVIF and PVF, an irregular cardiac rhythm hinders the collection of Doppler signals of the beat with same R-R interval. Thus, in atrial fibrillation, the beat with same R-R interval should be selected. When the simultaneous recording of LVIF and PVF is possible, this problem will be solved.

\section{Conclusions}

We developed a new algorithm to obtain the FVC and from the indices of FVC we can demonstrate that in patients with an abnormal relaxation pattern, the LA reservoir and booster pump functions are both augmented and may compensate for impaired LV diastolic function; however, in patients with a pseudonormal pattern, LA reservoir and booster pump functions are both impaired. This dysfunction of LA may be related to an increase in ANP and subsequent vulnerability to pulmonary congestion.

\section{References}

1. Braunwald E, Frahm CJ: Studies on Starling's law of the heart. IV. Observations on the hemodynamic functions of the left atrium in man. Circulation 1961; 24: 633-642

2. Swan HJC, Ganz W, Forrester JS, Marcus H, Diamond G, Chonette D: Catheterization of the heart in man with use of a flow-directed balloon-tipped catheter. N Engl J Med 1970; 283: 447-451

3. Bruns HA, Brode P: Left atrial function studied by cineangiocardiographic volume determinations. I. Studies with casts of normal human atria. Am J Roentgenol Radium Ther Nucl Med 1970; 110: 462-466

4. Bruns HA: Left atrial function studied by cineangiocardiographic volume determinations. II. Clinical application. Am J Roentgenol Radium Ther Nucl Med 1970; 110: 467-473

5. Matsuda Y, Toma Y, Ogawa H, Matsuzaki M, Katayama K, Fujii T, et al: Importance of left atrial function in patients with myocardial infarction. Circulation 1983; 67: 566-571

6. Toma Y, Matsuda Y, Moritani K, Ogawa H, Matsuzaki M, Kusukawa $\mathrm{R}$ : Left atrial filling in normal human subjects: Relation between left atrial contraction and left atrial early filling. Cardiovasc Res 1987; 21: $255-259$

7. Yamaguchi M, Arakawa M, Tanaka T, Takaya T, Nagano T, Hirakawa S: Study on left atrial contractile performance: Participation of Frank-Starling mechanism. Jpn Circ J 1987; 51: 1001 -1009

8. Matsuzaki M, Tamitani M, Toma Y, Qgawa H, Katayama K, Matsuda Y, et al: Mechanism of augmented left atrial pump function in myocardial infarction and essential hypertension evaluated by left atrial pressure-dimension relation. Am J Cardiol 1991; 67: 1121 1126

9. Glant C, Bunnell IL, Greene DG: The reservoir function of the left atrium during ventricular systole. Am J Med 1964; 37: 36-43

10. Payne RM, Stone HL, Engelken EJ: Atrial function during volume loading. J Appl Physiol 1971; 31: 326-331

11. Kawakami H, Sumimoto T, Matsuoka H, Kobayashi T, Ohtani T, Abe M, et al: Atrial natriuretic peptide and left atrial systolic function in normal subjects. Angiology 1993; 44: 903-907

12. Globits S, Frank H, Pacher B, Huelsmann M, Ogris E, Pacher R: Atrial natriuretic peptide release is more dependent on atrial filling volume than on filling pressure in chronic congestive heart failure. Am Heart J 1998; 135: 592-597

13. Keren G, Meisner JS, Sherez J, Yellin EL, Laniado S: Interrelationship of mid-diastolic mitral valve motion, pulmonary venous flow, and transmitral flow. Circulation 1986; 74: 36-44

14. Miyaguchi K, Iwase M, Yokota M, Hayashi H: Dependency of the pulsed Doppler-derived transmitral filling profile on the sampling site. Am Heart J 1991; 122: $142-148$

15. Appleton CP, Jensen JL, Hatle LK, Oh JK: Doppler evaluation of left and right ventricular diastolic function: A technical guide for obtaining optimal flow velocity recordings. J Am Soc Echocardiogr 1997; 10: $271-292$

16. Arakawa M, Miwa H, Noda T, Ito Y, Kagawa K, Nishigaki K, et al: Usefulness of the pulmonary vein flow velocity-time profile as an estimate of left atrial storage fraction. Angiology 1994; 45: 841-849 
17. Kitabatake A, Inoue M, Asao M, Tanouchi J, Masuyama T, Abe H, et al: Transmitral blood flow reflecting diastolic behavior of the left ventricle in health and disease: A study by pulsed Doppler technique. Jpn Circ J 1982; 46: $92-102$

18. Bough EW, Gandsman EJ, Shulman RS: Measurement of normal left atrial function with gated radionuclide angiography. Am J Cardiol 1981; 48: $473-478$

19. Wayne R: NIH image 1.61 manual. 1997. (Available at http://rsb.info. nih.gov/nih-image/manual/contents.html.)

20. Strunk BL, Fitzgerald JW, Lipton M, Popp RL, Barry WH: The posterior aortic wall echocardiogram: Its relationship to left atrial volume change. Circulation 1976; 54: 744-750

21. Biamino G, Wesel HJ, Schlag W, Schroeder R: Echocardiographic pattern of motion of the aortic root as a correlate of left atrial volume changes. Am Heart J 1980; 100: 191-200

22. Usui M, Asou T, Okada M, Arakawa M, Hirakawa S: Human adult left atrial volume estimated by M-mode echocardiography. J Cardiogr 1985; 15: 773 - 785 (in Japanese with English abstract)

23. Rossvoll O, Hatle LK: Pulmonary venous flow velocities recorded by transthoracic Doppler ultrasound: Relation to left ventricular diastolic pressures. J Am Coll Cardiol 1993; 21: 1687-1696
24. Nishimura RA, Tajik AJ: Evaluation of diastolic filling of left ventricle in health and disease: Doppler echocardiography is the clinician's Rosetta Stone. J Am Coll Cardiol 1997; 30: 8-18

25. Giannuzzi P, Imparato A, Temporelli PL, de Vito F, Silva PL, Scapellato F, et al: Doppler-derived mitral deceleration time of early filling as a strong predictor of pulmonary capillary wedge pressure in postinfarction patients with left ventricular systolic dysfunction. $J$ Am Coll Cardiol 1994; 23: 1630-1637

26. Castello R, Pearson AC, Lenzen P, Labovitz AJ: Evaluation of pulmonary venous flow by transesophageal echocardiography in subjects with a normal heart: Comparison with transthoracic echocardiography. J Am Coll Cardiol 1991; 18: 65-71

27. Rajagopalan B, Bertram CD, Stallard T, Lee GdeJ: Blood flow in pulmonary veins: III. Simultaneous measurements of their dimensions, intravascular pressure and flow. Cardiovasc Res 1979; 13: 684-692

28. Ormiston JA, Shah PM, Tei C, Wong M: Size and motion of the mitral valve annulus in man: A two-dimensional echocardiographic method and findings in normal subjects. Circulation 1981; 64: $113-$ 120 